\title{
Decoherence and dephasing in spin-based solid state quantum computers
}

\author{
Xuedong Hu, Rogerio de Sousa, and S. Das Sarma \\ Department of Physics, University of Maryland, College Park, MD 20742-4111
}

(February 1, 2008)

\begin{abstract}
We discuss the operational definition of decoherence in various solid state systems. In particular, we review, in the context of spin-based solid state quantum computation, the introduction of $T_{1}$ and $T_{2}$ to describe decoherence in a two level system. We provide a perspective on recent experiments involving the manipulation of spin coherence in semiconductors, and discuss specific decoherence and dephasing issues in electron spin-based quantum dot quantum computer architectures.
\end{abstract}

\section{INTRODUCTION}

A quantum eigenstate of a particular Hamiltonian is by definition a stationary state, in which the wavefunction might vary spatially, but does not decay in time. A quantum system can be in a superposition of its eigenstates with definite phase and amplitude relationships among the basis states. Such a superposition of eigenstates with definite phase relationships is called quantum coherence. Decoherence refers loosely to how a system loses these quantum coherence features. For example, it can refer to the amplitude decay (often exponential) and the associated disappearance of a quantum eigenstate in time (by virtue of it interacting with a surrounding bath, for instance). Or it may refer to the loss of electron phase coherence, because the definite phase relationship between the superposing states disappears over time leading to dephasing.

In an isolated quantum system decoherence could only arise from the dynamical degrees of freedom neglected in the original Hamiltonian used to define the quantum state. In a system coupled to an external bath, decoherence could arise naturally from the coupling between the system and the bath as the quantum eigenstate (presumably slowly) leaks to the environment (the bath) due to energy exchange between the system and the bath. Note that the bath or the environment does not need to be physically separated from the "system" - it is a standard practice in physics to divide a large system into sub-systems which are "reasonably isolated" (in some well-defined operational sense) from each other, i.e. the interaction Hamiltonian coupling the various sub-systems is "weak" in a precisely defined manner. In situations like this (which are commonplace in condensed matter physics) the "system" and the "bath" can be two components of the same system (such as electrons and phonons of a crystal lattice).

Decoherence in quantum mechanics has received a great deal of recent attention in the context of current interest in quantum computation and information processing. In a quantum computer (QC), an algorithm is typically performed by applying unitary operations on an array of two level systems (qubits) that carry quantum information [1]. For quantum computation, these qubits must be isolated from the other degrees of freedom that might perturb their unitary time evolution. In other words, decoherence in a quantum computer must be much slower than a typical quantum gate operation for successful quantum computation. The ratio between gate time and decoherence time needs to be smaller than $10^{-3} \sim 10^{-6}$ [1], which is the current quantum error correction limit. Thus the control of decoherence is a crucial aspect of quantum information processing. Decoherence is not only pertinent to performing quantum computation but is also a central issue in describing the transition between regimes governed by quantum mechanics and classical mechanics [2]. In fact, decoherence is a fundamental concept in the quantum measurement theory and in works dealing with the foundations of quantum mechanics.

In condensed matter physics, terminologies such as decoherence, dephasing, relaxation, scattering, etc, are often used quite liberally (and somewhat confusingly) due to the diversity of physical systems and phenomena studied. With the many existing proposals for solid state quantum computer architectures [4-33], there is obviously a need to accurately understand decoherence issues in each particular scheme. The specific decoherence channels are always system dependent, but there also exist many common features. For example, most QC proposals involve quantum two level systems (TLS) [34] serving the role of qubits. These TLS might be electron spin levels, electron orbital levels, nuclear spin levels, charging levels, magnetic flux directions, etc. The basic QC algorithm in each scheme involves dynamic manipulations of these TLS using various external means in order to perform one and two qubit operations. It is therefore imperative that the decoherence time in these TLS dynamics is much larger than the qubit operation times. Because of the two-level nature of these systems, it is possible to describe their decoherence using just two dephasing time scales: namely $T_{1}$ and $T_{2}\left(\leq T_{1}\right)$, which give a phenomenological description of the population and phase relaxation in these systems. For an ensemble of TLS, another time scale $T_{2}^{*} \leq T_{2}$ should also be defined, since some spins may rotate faster than others leading to reversible loss of quantum coherence between them. These two time scales $\left(T_{2}^{*}\right.$ and $\left.T_{2}\right)$ should be carefully distinguished, as the current QC proposals mostly involve single TLS so that $T_{2}$ is the relevant quantity, while in macroscopic measurements the observed quantity is often $T_{2}^{*}$ due to the 
ensemble averaging over the response from a large number of TLS. We should note that using just two relaxation times almost never gives a complete description of the dynamics and lineshapes of a realistic two-level system coupled to a physical environment. However, in several paradigmatic TLS such as nuclear spins probed by NMR (nuclear magnetic resonance) or electron spins probed by ESR (electron spin resonance), relaxation constants $T_{1}$ and $T_{2}$ give the proper qualitative description of the signal linewidths, and are good operational representations of the various relaxation channels. We also note that in many situations of interest $T_{1}$ and $T_{2}$ could be equal (or quite close in values).

In this paper we first review and discuss the definitions and significance of $T_{1}, T_{2}$, and $T_{2}^{*}$ in the context of macroscopic Bloch equations and the density matrix representation. We then review the role of these relaxation times in experiments involving semiconductors, which are projected to be of potential use in several proposed solid state QC architectures. We also discuss some specific features of decoherence in a solid state quantum computer, particularly aspects of gate induced decoherence in the form of non-adiabaticity, electrical circuit noise, and field inhomogeneity. We conclude with a brief discussion of electronic decoherence in mesoscopic systems.

\section{DECOHERENCE AND THE BLOCH EQUATIONS}

As decoherence occurs in the time domain, it is natural to use time scales to describe how strong a decoherence channel is, and how strongly decoherence affects a particular dynamic variable. If we restrict ourselves to a simple single TLS, the problem of describing phenomenologically the effect of weak coupling to external degrees of freedom on the time evolution of this TLS contains only a few parameters. In particular, two time scales of relaxation, $T_{1}$ and $T_{2}$, were introduced and used extensively in the field of NMR [35], and then used naturally also in ESR [36] and in quantum optics [37], in which either the applied static magnetic field (which causes Zeeman splitting along the field direction) or the natural TLS (e.g. for photons, where the longitudinal and transverse polarization occurs naturally) defines a longitudinal and a transverse direction. $T_{1}$ and $T_{2}$ are then respectively the longitudinal and transverse relaxation times for magnetizations in NMR and ESR, or the population difference and polarization in quantum optics. Note that using $T_{1}$ and $T_{2}$ to characterize decoherence applies only to TLS dynamics.

The definition of $T_{1}$ and $T_{2}$ is quite system specific - in fact, the strict definition of $T_{1}$ and $T_{2}$ apply specifically to magnetic resonance measurements. An arbitrary decoherence phenomenon might require more or less parameters to describe the dephasing process. In general, in the absence of magnetic field and in isotropic systems, $T_{1}=T_{2}$. In weak localization related mesoscopic experiments, a single electron dephasing time, which parametrizes the loss of quantum interference, is sufficient to characterize the results. We would also like to point out that $T_{1}$ and $T_{2}$ are purely phenomenological parameters (characterizing longitudinal and transverse relaxation respectively), to which many different decoherence mechanisms could, in principle, contribute. In general, two parameters may not be adequate to completely describe TLS decoherence, but experience (particularly in NMR, ESR, and optical pumping experiments) suggests that $T_{1}$ and $T_{2}$ are often quite sufficient in characterizing TLS decoherence in many diverse situations and are therefore extremely important TLS parameters.

We first discuss how $T_{1}$ and $T_{2}$ are introduced for a particular TLS - an electron spin in an external magnetic field. Consider an electron subject to a constant field in the $\mathrm{z}$ direction $\mathbf{B}_{\|}=B_{\|} \hat{\mathbf{z}}$, and a rotating field in the xy plane with frequency $\nu$ which perturbs its spin precession, $\mathbf{B}_{\perp}=B_{\perp}[\cos (\nu t) \hat{\mathbf{x}}+\sin (\nu t) \hat{\mathbf{y}}]$. The magnetic moment of the electronic spin is given by

$$
\vec{\mu}=\frac{g e}{2 m c} \mathbf{S}=-\frac{g}{2} \mu_{e} \vec{\sigma}
$$

where $\mu_{e}$ is the Bohr magneton, $g$ is the electron gyromagnetic ratio, and $\vec{\sigma}=\sigma_{x} \hat{\mathbf{x}}+\sigma_{y} \hat{\mathbf{y}}+\sigma_{z} \hat{\mathbf{z}}$ is the Pauli spin vector. The spin Hamiltonian $H=-\vec{\mu} \cdot \mathbf{B}$ is given by its parallel and perpendicular parts, $H=H_{\|}+H_{\perp}$ :

$$
H=-\vec{\mu} \cdot\left(B_{\perp}[\cos (\nu t) \hat{\mathbf{x}}+\sin (\nu t) \hat{\mathbf{y}}]+B_{\|} \hat{\mathbf{z}}\right)=\frac{1}{2}\left(\begin{array}{cc}
\hbar \omega & 2 \Delta e^{-i \nu t} \\
2 \Delta e^{i \nu t} & -\hbar \omega
\end{array}\right)
$$

where $\hbar \omega=g \mu_{e} B_{\|}$and $\Delta=\frac{g}{2} \mu_{e} B_{\perp}$. The spin dynamics under Hamiltonian (2) is governed by the von Neumann equation for the density matrix,

$$
i \hbar \dot{\rho}=[H, \rho] .
$$

We can remove the time dependence of $H$ by going to the interaction picture rotating in phase with $\mathbf{B}_{\perp}$, in which the equation of motion is much simplified. Since $\rho_{\uparrow \uparrow}+\rho_{\downarrow \downarrow}=1$ and $\rho_{\uparrow \downarrow}=\rho_{\downarrow \uparrow}^{*}$, only two equations describe the dynamics in the interaction picture: 


$$
\begin{aligned}
& i \hbar \dot{\rho}_{\uparrow \uparrow}=\Delta\left(\rho_{\uparrow \downarrow}^{*}-\rho_{\uparrow \downarrow}\right), \\
& i \hbar \dot{\rho}_{\uparrow \downarrow}=\hbar \delta \rho_{\uparrow \downarrow}+\Delta\left(\rho_{\downarrow \downarrow}-\rho_{\uparrow \uparrow}\right) .
\end{aligned}
$$

where $\delta=\omega-\nu$ is the detuning frequency of the applied transverse rotating field.

The evolution of this rotating or precessing electron spin is unitary since up to now we are considering a single isolated spin without any dephasing or decoherence. Quantum coherence is always maintained. However, an electron spin is never isolated. It couples to the electron orbital degrees of freedom through spin-orbit coupling, to nuclear spins through hyperfine interaction and dipole interaction, to the crystal lattice (and thus phonons) through spin-orbit coupling, to any magnetic impurities in the environment through direct spin dipole coupling, and to other electron spins through dipole and exchange coupling. With all these "environmental" degrees of freedom being present in principle, the simple decoherence-free equations for the spin density matrix above are obviously an idealization. To determine how strong these "outside" influences are, we need to include them in the starting Hamiltonian, and then employ approaches such as master equations or Langevin equations in order to achieve a quantitative understanding of how the system evolves and how the spin we are concerned with loses its coherence due to its coupling to the "bath", which, in this case, implies the collection of all the degrees of freedom neglected in our Hamiltonian of Eq. (2). This is obviously a very complicated problem (in fact, an insoluble problem since we can never be sure of all the possible degrees of freedom in the "bath"). A simple approach to tackle this problem is to add exponential decay terms to the right hand sides of the two equations above: $-\frac{i \hbar}{T_{1}} \rho_{\uparrow \uparrow}$ to Eq. (蛋) and $-\frac{i \hbar}{T_{2}} \rho_{\uparrow \downarrow}$ to Eq. (5) to mimic decoherence phenomenologically. This is similar to adding a friction term proportional to velocity in the classical Newton's equation, and is essentially a Markovian approximation to spin relaxation as it neglects memory effects in the system-environment coupling. The two time constants can be calculated under certain conditions if sufficient information about the bath is available. This simple phenomenological approach turns out to be quite successful in describing many experiments, ranging from NMR and ESR to quantum optics, although actual explicit calculations of $T_{1}$ and $T_{2}$ are generally quite difficult. It is perhaps more fruitful to consider $T_{1}$ and $T_{2}$ as purely phenomenological parameters (characterizing longitudinal and transverse relaxations respectively in the TLS dynamics) to be obtained from experimental measurements.

Recall that the unit magnetization vector is related to the spin via $\mathbf{M}=\operatorname{Tr}\left(\rho_{I} \vec{\sigma}\right)$, so that $M_{x}=2 \operatorname{Re}\left(\rho_{\uparrow \downarrow}\right)$, $M_{y}=-2 \operatorname{Im}\left(\rho_{\uparrow \downarrow}\right), M_{z}=\left(\rho_{\uparrow \uparrow}-\rho_{\downarrow \downarrow}\right)$. We can now rewrite Eqs. (4) and (5) with respect to the components of the real vector $\mathbf{M}$, obtaining the Bloch equations in the interaction picture [37,38]:

$$
\begin{aligned}
& \dot{M}_{x}=-\frac{1}{T_{2}} M_{x}-\delta M_{y}, \\
& \dot{M}_{y}=\delta M_{x}-\frac{1}{T_{2}} M_{y}+R_{0} M_{z}, \\
& \dot{M}_{z}=-R_{0} M_{y}-\frac{1}{T_{1}}\left(M_{z}+1\right),
\end{aligned}
$$

where $R_{0}=-2 \Delta / \hbar=-g e_{0} B_{\perp} /(m c)$ is the Rabi flopping frequency, negative for a free electron, whose magnetization rotates counter-clockwise in the yz plane (we keep this sign for conventional purposes). We readily see that $T_{2}$ is the relaxation time for the xy (transverse) magnetization of the electrons, whereas $T_{1}$ is the decay time for the $\mathrm{z}$ direction (longitudinal) magnetization. To describe an ensemble of spins, which may in general possess different Zeeman splittings $\hbar \omega$ (for example, by virtue of inhomogeneities in the applied magnetic field and/or in the electron g-factor) and thus having different detunings $\delta$ in the rotating field, additional ensemble averaging needs to be performed. This averaging leads to a different time constant $T_{2}^{*}\left(\leq T_{2}\right)$ to describe the width of the magnetic resonance signal (the inhomogeneous broadening), but it does not affect the longitudinal direction. Note that $T_{2}$ (or $T_{2}^{*}$ ) describes the dephasing process ( $T_{2}$ is often called the dephasing time), and $T_{1}\left(\geq T_{2}\right)$ is the inelastic spin-flip or spin-lattice relaxation time. Often $T_{2}$ is also called the spin-spin relaxation time for reasons to be discussed below. The Bloch equations successfully describe dephasing and relaxation phenomena in atoms, quantum optics, nuclear spins, and electron spins in semiconductors [35 33,.39], even though microscopically it may well be the case that only two time scales are not sufficient to describe the dynamics, or that a continuous time description of the single spin magnetization is only appropriate as an average behavior, since the spin might be subjected to discrete random "kicks".

Two particular solutions of the Bloch equations are of special interest. If there is no pure dephasing process (which we will discuss below) in the system, $T_{1}=T_{2}$, i.e. all dephasing arises from spin-flip inelastic scattering. In this case the magnetization rotates at the generalized Rabi flopping frequency $R=\sqrt{R_{0}^{2}+\delta^{2}}$, and the equations for the magnetization can be written in a succinct vectorial form:

$$
\dot{\mathbf{M}}=-\frac{1}{T_{1}}(\mathbf{M}+\hat{\mathbf{z}})+\left(\delta \hat{\mathbf{z}}-R_{0} \hat{\mathbf{x}}\right) \times \mathbf{M} .
$$


In general, $T_{1} \neq T_{2}$, and Eq. (9) does not apply. Without the external tipping field, so that $R_{0}=0$, the system undergoes a so-called free induction decay [35] 37] if it is initially pushed out of equilibrium:

$$
\dot{\mathbf{M}}=-\frac{1}{T_{2}} \mathbf{M}_{\perp}+\omega \hat{\mathbf{z}} \times \mathbf{M}_{\perp}-\frac{1}{T_{1}}\left(M_{z}+1\right) \hat{\mathbf{z}} .
$$

The density matrix takes on the form of Boltzmann distribution at large time $\left(t \gg T_{1}\right)$ and finite temperature:

$$
\rho(t)=\left(\begin{array}{cc}
\left(\rho_{\uparrow \uparrow}(0)-a_{0}\right) e^{-\frac{t}{T_{1}}}+a_{0} & \rho_{\uparrow \downarrow}(0) e^{-i \omega t} e^{-\frac{t}{T_{2}}} \\
\rho_{\uparrow \downarrow}^{*}(0) e^{i \omega t} e^{-\frac{t}{T_{2}}} & \left(a_{0}-\rho_{\uparrow \uparrow}(0)\right) e^{-\frac{t}{T_{1}}}+1-a_{0}
\end{array}\right),
$$

where $a_{0}=e^{-\beta \mu_{e} B} /\left(e^{-\beta \mu_{e} B}+e^{\beta \mu_{e} B}\right)$ is the thermal partition function at equilibrium with $\beta=\left(k_{B} T\right)^{-1}$ as usual (where $T$ is the absolute temperature, not a relaxation time).

Regarding the decoherence of a single spin, we note that spin-flip processes cause both population relaxation and dephasing, contributing to both rates $1 / T_{1}$ and $1 / T_{2}$. However, in a real physical system the longitudinal and transverse directions are often affected differently by the environment. Indeed, there exist pure dephasing processes which affect only $T_{2}$ but not $T_{1}$. One example is the colliding molecules in an optically active gaseous medium, where molecules constantly undergo collisions with each other, some of them inelastic, but most of them elastic. During an elastic collision, the single molecule energy spectrum changes due to the presence of the other molecule nearby. This shift in energy levels (particularly the two active levels) is dependent on the details of the collision, and is thus a random variable, which we refer to as $\delta \omega(t)$. Including this frequency shift the differential equation (5) for $\rho_{\uparrow \downarrow}$ becomes (here we are using the spin language to describe an orbital TLS, with spin up and down referring to the two levels)

$$
\dot{\rho}_{\uparrow \downarrow}(t)=-i(\omega+\delta \omega(t)) \rho_{\uparrow \downarrow} .
$$

Note that in the first order approximation $\rho_{\uparrow \uparrow}$ is not affected since its equation of motion (4) is independent of $\omega$. $\delta \omega(t)$ is a random variable that averages to zero, $\langle\delta \omega(t)\rangle=0$. In the Markovian approximation (no memory effects in the random variation of $\delta \omega(t))$ we have

$$
\left\langle\delta \omega(t) \delta \omega\left(t^{\prime}\right)\right\rangle=2 \gamma_{p h} \delta\left(t-t^{\prime}\right) .
$$

This random fluctuation in energy level splitting of the TLS then causes a pure dephasing effect:

$$
\left\langle\rho_{\uparrow \downarrow}(t)\right\rangle=\left\langle\rho_{\uparrow \downarrow}(0)\right\rangle e^{-i \omega t} e^{-\gamma_{p h} t} .
$$

This pure dephasing only contributes to $T_{2}$, but not to $T_{1}$. Another well-known example of pure dephasing is the dipolar spin-spin interaction in NMR, which produces effective local magnetic field fluctuations and hence contributes essentially only to $T_{2}$ (the corresponding effect on $T_{1}$ is extremely small). What is important for dephasing is that some change in the state of the environment must occur due to its interaction with the system - dephasing does not necessarily require an explicit inelastic scattering process for the system, although all inelastic scatterings necessarily produce dephasing. In fact, as mentioned before, $T_{2}$ in the context of ESR and NMR is often called the spin-spin relaxation time because the most important intrinsic effect contributing to $1 / T_{2}$ is the dipolar interaction among various spins in the system, which, while transferring energy among the spins themselves, does not lead to overall energy relaxation from the total spin system. By contrast, spin-lattice interactions lead to energy relaxation (via spin-flip processes) from the spin system to the lattice, and thus contribute to $T_{1}^{-1}$, the spin-lattice relaxation rate. We note in this context that $T_{2}$ sets the time scale for the spin system to achieve equilibrium within itself whereas $T_{1}$ sets the time scale for the global thermodynamic equilibrium between the spin system and the lattice. It should be emphasized that all inelastic processes contributing to $T_{1}$ also automatically lead to dephasing, but in many circumstances there may be additional dephasing processes (e.g. dipolar spin-spin coupling in NMR and ESR) which contribute only to $T_{2}$ (and not to $T_{1}$ ), and therefore $T_{1} \geq T_{2}$ in general.

\section{MEASURING $T_{1}, T_{2}$, AND $T_{2}^{*}$ OF ELECTRON SPINS IN SEMICONDUCTORS}

In metals and doped semiconductors there are three major spin relaxation mechanisms for conduction electrons 40,41 except at the lowest temperatures, where relaxation times are extremely long and dipolar and nuclear coupling become comparatively important. In the Elliot-Yafet mechanism, spin-independent interactions of electrons with impurities, boundaries, interfaces, and phonons connect up and down spins through spin-orbit coupling. In the 
Dyakonov-Perel' mechanism, the splitting of spin up and down conduction bands due to lack of inversion symmetry (as in III-V semiconductors which have the zinc-blende lattice structure) acts as an effective momentum dependent magnetic field $\mathbf{B}(\mathbf{k})$. An electron with momentum $\mathbf{k}$ and spin $\mathbf{S}$ precesses in this effective field $\mathbf{B}(\mathbf{k})$ and loses its spin memory. As this electron is scattered into a different $\mathbf{k}$ state, its spin will start to precess around the new effective field. This constant change of effective magnetic field actually impedes the electron spin relaxation, so that the spin relaxation time is inversely proportional to the momentum relaxation time in this mechanism. The third mechanism, the Bir-Aronov-Pikus mechanism, is given by the exchange interaction between electrons and holes. Electronic spins move in an effective field produced by the hole spins, and relaxation takes place when hole spins change in a rate much faster than the electron precession frequency. A more detailed review of these mechanisms and their experimental observations in metals and semiconductors have been discussed in reference [11]. We only mention here that in general electron spin relaxation in GaAs is weak due to its relatively weak conduction band spin-orbit coupling - a simple matrix element estimate indicates that the spin relaxation time is of the order of 10-100 ns at low temperatures ( $T \sim 4 \mathrm{~K}$ ), and one should approximately have $T_{1} \sim T_{2}$ in high quality GaAs. Such a "long" relaxation time $\left(T_{2}\right)$ has recently been directly measured in ESR experiments [42]. We should emphasize that this relaxation time $(\sim 10-100$ $\mathrm{ns})$ is "long" only in a relative sense compared with electron-electron scattering times $(\sim \mathrm{fs})$ or momentum relaxation times $(\sim$ ps $)$ - electron spin relaxation in GaAs should be rather slow due to very weak conduction band spin-orbit coupling, and the observed relaxation times are not long in any absolute sense. In fact, low temperature electron spin relaxation times in metals are also very long $(\sim \mu \mathrm{s})$ [11.

Coherent manipulation of electron spins and the study of electron spin relaxation have a long history going back to the first ESR experiments half a century ago [36], and ESR remains a key technique to determine electron spin relaxation and study electron spin dynamics [42. Below we focus on some of the recent experiments that employ alternative optical approaches to study electron spins in semiconductors, especially on optical orientation and related measurements of spin relaxation, which may have particular relevance to solid state quantum computation, where externally controlled optical pulses may be used to coherently manipulate electron spin dynamics in zinc-blende semiconductor structures.

\section{A. Bulk III-V semiconductors}

The method of optical orientation can be used to measure spin relaxation $\left(T_{1}\right)$ in bulk III-V semiconductors like GaAs 40]. Shining circularly polarized light on a semiconductor generates polarized conduction electrons with spin oriented in the direction of the light beam. These spins will relax until the electrons recombine with holes in a typical time $\tau_{p}$ when a photon is emitted. If $\tau_{p} \ll T_{1}$, then the recombination photon will also be circularly polarized. Since $\tau_{p}$ can be extracted by other techniques one can measure $T_{1}$ by studying the polarization of the recombination luminescence. If a magnetic field is applied perpendicular to the spin orientation of the electrons, the recombination radiation is less polarized (Hanlé effect). This effect can be used to measure both $\tau_{p}$ and $T_{1}$ [40]. Typical values for $T_{1}$ in p-doped GaAs are 10 ps to $1 \mathrm{~ns}$, depending on the sample temperature and impurity concentration. Thus, the hole spin relaxation rate is relatively fast in GaAs, consistent with its strong valence band spin-orbit coupling.

Optical measurements have recently been used to measure $T_{2}^{*}$ in n-doped samples of bulk GaAs, using pump-probe and Faraday rotation techniques [43]. A circularly polarized laser pulse is used to pump electrons to the conduction band. The holes left behind by the excited electrons quickly lose their spin polarization (due to strong valence band spin-orbit coupling for holes) and are annihilated by the doped electrons in the first $100 \mathrm{ps}$ after the pump pulse, substantially reducing the hole-related spin relaxation (Bir-Aronov-Pikus mechanism). A linearly polarized laser pulse is then used to probe the remaining electron spins a time $\Delta t$ after the pump pulse. When this pulse scatters off the partially polarized electrons, its linear polarization is rotated by an angle $\theta$ proportional to the projection of the spin magnetization in the direction of the laser beam, a phenomenon known as Faraday rotation. By using pump and probe pulses of about $100 \mathrm{fs}$ in duration respectively, the time resolved Faraday rotation technique can reveal the time dependence of the magnetization of the spins in the $\mathrm{x}$ direction,

$$
M_{x}=M_{x}(\Delta t=0) e^{-\frac{\Delta t}{T_{2}^{*}}} \cos \left(g \mu_{e} B \Delta t / \hbar\right),
$$

with $\Delta t$ being the time delay between pump and probe pulses. At $B=1 \mathrm{~T}, T=5 \mathrm{~K}$, and doping concentration $n=10^{16} \mathrm{~cm}^{-3}$, a spin decoherence time $T_{2}^{*}$ of approximately $10 \mathrm{~ns}$ was measured [43]. Furthermore, $T_{2}^{*}=130 \mathrm{~ns}$ is obtained at $B=0$, which is one order of magnitude larger than the value at $B=1 \mathrm{~T}$. Since the spins are not precessing at $B=0$, one could argue that in this particular case $T_{2}^{*}=T_{2}=T_{1}=130 \mathrm{~ns}$, which is the decay of the magnetization in all direction since there is no magnetic field to break the symmetry, hence the increase by one order of magnitude (note that without an external magnetic field, the g factor inhomogeneities discussed in [43.44] will not 
contribute to $T_{2}^{*}$ ). This value of the relaxation time is consistent with the direct ESR measurement of the linewidth to be around $50 \mathrm{MHz}$ in GaAs heterostructure 42].

Time-resolved Faraday rotation was also used to study spin precession in an ensemble of chemically synthesized CdSe quantum dots 44]. $T_{2}^{I n h}$ (which contains averaging not only for the many electrons in one quantum dot (QD), but also over many quantum dots) was measured ranging from 3 ns at $B=0$ to less than $100 \mathrm{ps}$ at $B=4 \mathrm{~T}$. It was suggested that this strong field dependence comes from the fact that the quantum dots have varying $\mathrm{g}$ factors ranging between 1.1 and 1.7. To measure the decoherence time $T_{2}^{*}$ of the many electrons in a single quantum dot one could in principle use the spin echo technique to constantly realign the spins and observe the time decay of the transverse magnetization, hence extracting $T_{2}^{*}$ from the data of an ensemble of quantum dots. This experiment has not yet been performed, although the tipping technique required by spin echo type of experiments has recently been demonstrated [45].

\section{B. Single spin in a quantum dot}

Based on the assumption that an electron spin possesses long coherence times $\left(T_{1}, T_{2}\right.$ much greater than the time to perform qubit operations, see below), a spin-based quantum dot quantum computer (QDQC) has been proposed [3]. It is thus desirable to have estimates of $T_{1}$ and $T_{2}$ for single electron spins in a quantum dot, and to confirm the estimates experimentally. It is important to mention in this context that the single spin decoherence times should be longer than the macroscopic (averaged over many spins) values of $T_{1}$ and $T_{2}$, which should thus serve as an upper limit.

Phonon-assisted spin flip rates due to spin-orbit coupling in a single electron GaAs QD has been calculated [46]. It was pointed out that, due to wave function localization, the spin orbit relaxation mechanisms discussed above for a free electron (Elliot-Yafet, Dyakonov-Perel') are strongly suppressed in a QD, giving a long spin flip time: $T_{1} \approx 1 \mathrm{~ms}$ for $B=1 \mathrm{~T}$ and $T=0 \mathrm{~K}$. It was further noticed that spin relaxation is dominated by the Elliot-Yafet mechanism, which yields $T_{1} \propto B^{-5}$ for transitions between Zeeman sublevels in a one electron QD.

These calculations are consistent with recent transport measurements [47]. Pulses of current were injected into a QD coupled to leads in the Coulomb Blockade regime, where the decay rate from excited states can be measured by analyzing the decay time of transient currents generated by the pulses. The results indicate that, for $T=150 \mathrm{mK}$ and $B=0-2 \mathrm{~T}$, spin relaxation times $\left(T_{1}\right)$ in a many-electron QD (less than 50 electrons) are longer than at least a few $\mu \mathrm{s}$. This is encouraging from the perspective of the spin-based solid state QC architecture where spin relaxation times of $\mu$ s or longer are most likely necessary for large scale QC operation.

ESR combined with transport techniques in principle could be used to probe $T_{2}$ in a QD in the Coulomb Blockade regime. It has recently been proposed [4] that by applying an AC pump field to a single electron QD subjected to a magnetic field, the stationary current through this QD will exhibit a peak as a function of the pump frequency, whose width will yield a lower bound on $T_{2}$. This is in essence an ESR measurement with a transport readout.

It is desirable that $T_{2}$ for an electron in a single QD is a factor of $10^{4}$ or so greater than the typical gating time in a QDQC [1]. For $B=1 \mathrm{~T}$, the Zeeman splitting in a QD is about $0.03 \mathrm{meV}$, which yields 100 ps for the precession time of one spin, which can be used as the one qubit gate (the two qubit gate time is lower, $\hbar / J \sim 50$ ps for $J \sim 0.1 \mathrm{meV}$ ). Therefore for quantum error correction to be performed reliably, $T_{2}$ for the trapped electron spin needs to be on the $\mu$ s time scale, which may very well be the case at low enough temperatures in a single QD. We note that the existing experimental estimates of free electron spin relaxation time $T_{2}$ (or $T_{2}^{*}$ ) in GaAs (for $T=1-4 \mathrm{~K}$ ) is around $10-100 \mathrm{~ns}$, which is obviously a lower bound since one expects on rather general ground the spin relaxation time of individual electrons confined in QD structures to be longer. Much of the current optimism in the feasibility of spin-based QDQC architecture arises from the expected long electron spin relaxation times (many $\mu$ s or longer at low temperatures) of GaAs electrons confined in high quality QDs.

\section{DECOHERENCE IN A QUANTUM DOT QUANTUM COMPUTER}

Defining a TLS involves choosing two levels that are well isolated from the rest of the degrees of freedom in the overall system. In a solid state system, where the density of states is generally high and where long range electromagnetic interaction typically couples all the charged entities, isolating two levels is particularly difficult. In a spin-based quantum computer, the spin up and down states are chosen as the two states of a qubit. It is then crucial to explore all relevant degrees of freedom in the solid state environment that couple to the electron spins and determine whether they are sufficiently weak (or can be made so by suitable means) for a quantum computer to work. 
Trapped in an ideal quantum dot in a perfect heterostructure, an electron is sitting at the bottom of the conduction band, and there should be no other electrons or holes nearby in order for the trapped electron to be considered as a candidate for qubit. Thus the DP and BAP spin relaxation mechanisms should not be important. The major environmental influences come from spin-orbit coupling (therefore phonons) and nuclear spins in the material, assuming dipolar coupling to other electron spins to be weak; otherwise dipolar coupling to other spins has to be accounted for as well. The experimental temperature can be lowered so that no resonant phonon (producing decoherence) is present in the system, and nuclear spins need to be controlled via various means. However, these controls are not sufficient because imperfections (e.g. impurities, defects, etc.) are facts of life in artificially created QD structures. Thus there are additional factors that need to be considered such as imperfect interfaces and nearby metallic gates as well as the associated charge diffusion and charge traps. Boundary scattering can probably be sufficiently suppressed by enhancing interface material quality and by making the quantum dots small. On the other hand, it is much harder to control charge transfers between the metallic gates and the heterostructure in which the quantum dots are located. Since the gates cannot be too far away from the dots (in order to produce small dots), any charge diffusion or trapping can lead to important (and often unknown) environmental effects. For example, if a stray electron is trapped too close to a quantum dot, there may be an exchange interaction between the electrons, which would cause complete loss of spin information in the quantum dot, destroying qubit operation.

When we bring two quantum dots close to each other in order to perform exchange gate operations required for quantum computing [3], there are additional external influences on the system. One concern is whether Heisenberg exchange Hamiltonian is a complete description of the low energy dynamics of a double dot. The higher excited states should certainly be eliminated (i.e. pushed to high energy) from the system, which we will discuss further below. Inherent spin-orbit coupling in 2D quantum dots leads to an anisotropic exchange, whose effects have to be controlled and, if necessary, corrected 49,50]. It is self-evident that the environmental influences for both single and double quantum dot structures will have to be carefully studied before a functional QDQC (even with just a few qubits) can be fabricated.

For a QC to work, we need to understand not only its full environment, but also how to best control it. To operate a QC, we inevitably need to manipulate the spins (or other form of qubits) using external means: gate voltages, applied magnetic fields, light, microwave, etc. When we introduce these external influences, we inevitably introduce unwanted perturbations. These can be called gate errors. Since these external perturbations necessarily involve some leakage of the system into its environment, they can also be regarded as a form of decoherence.

For example, in performing exchange gates in spin-based QDQC, the potential barrier between two neighboring quantum dots needs to be lowered to allow the electron wavefunctions to overlap and thus enabling the exchange interaction to take effect. However, the mixing of the two electrons naturally leads to the possibility of exciting the previously frozen orbital degrees of freedom, especially if the mixing happens too fast. It has been pointed out that adiabatic operation of the exchange gate can help suppress the errors caused by the state mixing [4.51]. Essentially, if the two-qubit system is initially prepared as a superposition of the ground singlet and triplet states, as long as the adiabatic condition is satisfied, the system will remain in the sub-space spanned by the ground singlet and triplet states, no matter how these states change during the barrier variation. Of course, the adiabatic condition can never be satisfied exactly in any real situation, therefore the important issue is to estimate the amount of mixing of the higher energy states under realistic conditions. We have recently done a calculation 52] of the time evolution of a two-electron double dot system when the central barrier between the dots is varied, so that we can quantitatively determine the adiabatic condition that a spin-based QDQC has to satisfy.

Under the adiabatic approximation, a quantum state can be expanded in the instantaneous basis of the system: $\psi(t)=\sum_{i} c_{i}(t) u_{i}(t)$, and the instantaneous basis states satisfy $H(t) u_{i}(t)=E_{i}(t) u_{i}(t)$. Thus the Schrödinger equation takes the form:

$$
\frac{\partial c_{k}}{\partial t}=\sum_{i \neq k}^{N} \frac{c_{i}}{E_{k}-E_{i}}\left\langle k\left|\frac{\partial H}{\partial t}\right| i\right\rangle \exp \left\{\frac{1}{i \hbar} \int_{-\infty}^{t}\left(E_{i}-E_{k}\right) d \tau\right\} .
$$

Here $N$ is the total number of basis states involved. To perform a simulation of the dynamics of this system, we need to know all the eigenstates and eigenenergies at all time. Since the eigenstates and energies vary relatively slowly with the change in the Hamiltonian, specifically the change in the barrier height between the two dots in our case, we did the eigensystem calculation on a finite grid, then obtained the rest of the information through interpolation. Our results show [52] that for a typical configuration [5] of the double dot system for quantum computing, the leakage rate would be reasonably small $\left(<10^{-6}\right)$ for gate operation times longer than 50 ps. Thus adiabatic condition is not overly stringent and should not hamper the operation of a QDQC. Note that the optimum value of the gate operation time is constrained from below by the adiabatic condition (i.e. gates should not be too "fast") and from above by the spin relaxation time (i.e. gates should be much faster than the typical spin relaxation time). Our most optimistic 
reasonable estimate of the applicable range of gating time for spin-based QDQC operations is 50 ps to 1 ns, which is not an unrealistic operation regime.

As the exchange coupling $J$ (the singlet-triplet splitting) is tuned [5] by changing external gate voltage in a QDQC, thermal fluctuations (or any other types of fluctuations) in the gate voltage will lead to fluctuations in $J$, thus causing phase errors in the exchange-based swap gate which is crucial for two-qubit operations. We have estimated this error by assuming a simple thermal (white) noise [5]. More specifically, we assume $J=f(V)$ where $V$ is the gate voltage that controls the value of $J$. Around any particular value $V_{0}, J$ can be expressed as $J(V)=$ $J\left(V_{0}\right)+\left.f^{\prime}(V)\right|_{V_{0}}\left(V-V_{0}\right)$. During a swap gate between two quantum dots, the phase of the electronic spin wavefunction evolves as $\phi=\int_{0}^{t} J(\tau) d \tau / \hbar$. Thus the fluctuation in the phase $\phi$ is

$$
\begin{aligned}
\left\langle\delta \phi^{2}\right\rangle & =\left\langle\phi^{2}\right\rangle-\langle\phi\rangle^{2}=\frac{1}{\hbar^{2}} \int_{0}^{t} \int_{0}^{t}\left\langle\delta J\left(\tau_{1}\right) \delta J\left(\tau_{2}\right)\right\rangle d \tau_{1} d \tau_{2} \\
& \sim \int_{0}^{t} \int_{0}^{t} \frac{\left[f^{\prime}(\bar{V})\right]^{2}}{\hbar^{2}}\left\langle\delta V\left(\tau_{1}\right) \delta V\left(\tau_{2}\right)\right\rangle d \tau_{1} d \tau_{2} .
\end{aligned}
$$

Using Nyquist theorem $\left\langle\delta V\left(\tau_{1}\right) \delta V\left(\tau_{2}\right)\right\rangle=4 R k_{B} T \delta\left(\tau_{1}-\tau_{2}\right)$, we obtain the approximate expression for the phase fluctuation:

$$
\left\langle\delta \phi^{2}\right\rangle \sim 4 R k_{B} T \alpha^{2} t / \hbar^{2},
$$

where $\alpha$ is the upper bound of $\left|f^{\prime}(\bar{V})\right|$. Assuming the swap gate is performed at $1 \mathrm{~K}$ (since $J$ is in the order of $0.1 \mathrm{meV}$ $\sim 1 \mathrm{~K}$, the experimental temperature cannot really be much higher than $1 \mathrm{~K}$ ), and the transmission line connecting the gate to the surrounding cryogenic bath has an impedance of $50 \mathrm{ohms}$, the rate for phase fluctuation $\left\langle\delta \phi^{2}\right\rangle / t$ is about 3.2 MHz. The phase error accrued during a swap gate is then about $0.06 \%$. This is quite a small error which is of the same order of magnitude as the theoretical tolerance of the currently available quantum error correction codes. To further lower this error rate, one can lower the experimental temperature and set up the gate architecture in such a way as to decrease the sensitivity of the exchange coupling $J$ on the gate voltage $V$ in the operation of a QDQC.

Another possible error in the two-qubit operations of the QDQC architecture is caused by inhomogeneous magnetic fields [53,54]. Such a field may come from magnetic impurities or unwanted currents away from the structure. Magnetic field affects both orbital and spin parts of the electron wavefunction. The orbital effect is accounted for by adjusted exchange coupling $J$, while the spin effect is accounted for through Zeeman coupling terms:

$$
H_{s}=J(\mathbf{B}) \mathbf{S}_{1} \cdot \mathbf{S}_{2}+\gamma_{1} S_{1 z}+\gamma_{2} S_{2 z},
$$

where $\mathbf{S}_{1}$ and $\mathbf{S}_{2}$ refer to the spins of the two electrons, $J(\mathbf{B})$ is the exchange coupling (singlet-triplet splitting), and $\gamma_{1}$ and $\gamma_{2}$ are the effective strength of the Zeeman coupling in the two quantum dots. In an inhomogeneous field, $\gamma_{1} \neq \gamma_{2}$, so that the Zeeman terms do not commute with the exchange term in the Hamiltonian (19). We have done a detailed analysis [53,54 on how to achieve swap with such a Hamiltonian, and found that there is at the minimum an error proportional to the square of field inhomogeneity in the swap. For example, if the initial state of the two electron spin is $|\phi(0)\rangle=|\uparrow \downarrow\rangle$, the density matrix of the first spin after the optimal swap is

$$
\left.\rho_{1}\right|_{e^{i \theta}=-1}=\frac{1}{1+x^{2}}|\downarrow\rangle\left\langle\downarrow\left|+\frac{x^{2}}{1+x^{2}}\right| \uparrow\right\rangle\langle\uparrow|,
$$

where $x=\delta / 2 J=\left(\gamma_{1}-\gamma_{2}\right) / 2 J$. In other words, the first spin can never exactly acquire the state $(|\downarrow\rangle)$ of the second spin. Its state will remain mixed and the smallest error from an exact swap is $x^{2} /\left(1+x^{2}\right)$, which needs to be corrected. We have estimated [53] that in GaAs a Bohr magneton can lead to an error in the order of $10^{-6}$, which is within the capability of currently available quantum error correction schemes.

In concluding this section, we mention that many of the techniques developed over the last fifty years in the context of NMR and ESR studies should be useful in controlling decoherence (and carrying out error corrections) in spinbased QDQC operations. These include the spin echo and refocusing techniques. In fact, our best estimates for the electron spin relaxation time in GaAs quantum dots $(\sim \mu s)$, the exchange coupling $(\sim 0.1 \mathrm{meV})$, and the gating time (ps to ns) makes us guardedly optimistic that a spin-based QDQC architecture may very well be developed in the future. It should, however, be kept in mind in this context that QC architectures [1] based on atomic physics (e.g. trapped ions) and liquid state NMR have extremely long natural decoherence times $\left(T_{2} \sim \mu \mathrm{s}-\mathrm{ms} ; T_{1} \sim\right.$ minutes-hours $)$ because of the extremely weak environmental coupling in these systems. The main problem in these architectures is not decoherence, but scaling up to more than a few (2-10) qubits which should be relatively easy in semiconductor solid state systems 23 . 


\section{MESOSCOPIC DECOHERENCE}

Finally, we provide a very brief discussion on the issue of electronic decoherence in mesoscopic electronic materials (e.g. metals, semiconductors). Our discussion is necessarily brief (done only in the context of quantum computing), and certainly will not do justice to the vast literature and the great deal of current activity in the subject. We provide this discussion only for the sake of completeness, restricting ourselves entirely to rather elementary considerations.

Electronic decoherence in mesoscopic materials is often characterized by dephasing (or the phase relaxation) time $\tau_{\phi}$ which shows up in electronic phase coherent processes (i.e. quantum interference phenomena) such as weak localization, conductance fluctuations, and various quantum interference oscillations (i.e. $h / e$ and $h / 2 e$ oscillations in connected structures). In general, each quantum coherent phenomenon may involve a slightly different (but closely related) definition of $\tau_{\phi}$, but in all cases $\tau_{\phi}$ indicates the typical dephasing time over which quantum interference memory is lost in the system [55]. The dephasing time $\tau_{\phi}$ in the many-body electronic system is similar in spirit to the dephasing time $T_{2}$ in the TLS case although, in contrast to TLS dynamics, the concept of a transverse relaxation time (as $T_{2}$ is in the TLS case) is not germane to the mesoscopic problem. The inelastic scattering (or relaxation) time $\tau_{i}$ (sometimes also called quasiparticle lifetime) in the electronic many-body system corresponds loosely to the inelastic spin-flip relaxation time $T_{1}$ in the TLS problem. Actually, the loose analogy between $T_{2}$ and $\tau_{\phi}$ on the one hand and $T_{1}$ and $\tau_{i}$ on the other hand could be further elucidated. Just as one of the main contributions to $1 / T_{2}$ at low temperatures is the spin-spin (dipolar) interaction, the main contribution to $1 / \tau_{\phi}$ at low temperatures is the electron-electron interaction. Similarly, if one restricts to energy loss from the whole electron system (and not just the individual electrons), then electron-phonon interaction is the main inelastic scattering mechanism in electronic systems, just as spin-lattice relaxation is the inelastic spin-flipping mechanism in $T_{1}$.

While $\tau_{i}$ is an inelastic lifetime related to the inverse of the one-electron self-energy, $\tau_{\phi}$ relates directly to the dephasing process. They are closely connected, but not necessarily the same, particularly at low temperatures and in low dimensional systems. In general, elastic scattering by impurities (where the impurities are considered static and immobile) does not directly contribute to either $\tau_{i}$ or $\tau_{\phi}$. The presence of impurity scattering does, however, have strong indirect influence on both $\tau_{i}$ and $\tau_{\phi}$, since the electronic motion in mesoscopic systems is diffusive (with a finite transport mean free path) in the presence of impurity scattering. For example, in a 3D metallic system the electron-electron scattering contribution (which is expected to dominate at low temperatures where the phonons are frozen out) to $\tau_{i}$ is $\tau_{i}^{-1} \sim T^{2}$ for a ballistic system, and $\tau_{\phi}^{-1}, \tau_{i}^{-1} \sim T^{3 / 2}$ in a diffusive system. Note that in a diffusive 3D system $\tau_{\phi} \sim \tau_{i}$ for electron-electron scattering (this is also true for electron-phonon scattering which is important at higher temperatures). This approximate equality of $\tau_{i}$ and $\tau_{\phi}$ for $3 \mathrm{D}$ systems follows primarily from the fact that large energy transfer $\left(\sim k_{B} T\right)$ scatterings dominate both $\tau_{i}$ and $\tau_{\phi}$ in 3D systems. In lower dimensional systems, however, this is not true in general, and we can have $\tau_{i} \gg \tau_{\phi}$ with $\tau_{\phi}^{-1}$ being dominated by very small energy scattering processes which in general do not much affect $\tau_{i}^{-1}$. The calculated temperature dependence of $\tau_{i}$ and $\tau_{\phi}$ due to electron-electron scattering in $2 \mathrm{D}$ diffusive systems are, however, the same up to a logarithmic correction: $\tau_{i}^{-1} \sim T \ln T, \tau_{\phi}^{-1} \sim T$. In 1D, on the other hand, $\tau_{\phi}^{-1} \sim T^{2 / 3}$ and $\tau_{i}^{-1} \sim T^{1 / 2}$. For electron-phonon scattering one typically finds [56] $\tau_{e-p h} \approx \tau_{\phi} \sim T^{-p}$ with $p \approx 1-4$ depending on systems and dimensionalities. Usually electronelectron scattering dominates $\tau_{\phi}$ for $T<10 \mathrm{~K}$ and electron-phonon scattering dominates at higher temperatures. It may be worthwhile to point out that just as the existence of $T_{2}$ (with $1 / T_{2} \neq 0$ ) is essential in defining a spin temperature $T_{s}$ (through spin-spin interaction) in ESR and NMR measurements [35, 36], electron-electron scattering is essential in defining an electron temperature $T_{e}$ in an excited electron gas. The final equilibrium to the lattice temperature $T_{L}$ (i.e. $T_{s} \rightarrow T_{L}$ or $T_{e} \rightarrow T_{L}$ ) is achieved in both cases through the interaction with the lattice (i.e. $1 / T_{1} \neq 0$ or $\left.1 / \tau_{e-p h} \neq 0\right)$.

The strong temperature dependence of $\tau_{\phi}\left(\sim T^{-3 / 2}\right.$ in $3 \mathrm{D}, T^{-1}$ in $2 \mathrm{D}$, and $T^{-2 / 3}$ in $\left.1 \mathrm{D}\right)$ at low temperatures is only observed over a rather limited range of temperature in experiments where the measured $\tau_{\phi}$ in many different (particularly, low dimensional mesoscopic) systems always seems to saturate at low enough temperature and cross over to a constant value of the order of $1 \mathrm{~ns}$ (with the crossover temperature of the order of $100 \mathrm{mK}$ ) [57]. Neither the saturation temperature nor the saturated value of $\tau_{\phi}$ seems to have any obvious universal behavior. Whether this low temperature $\tau_{\phi}$ saturation has any fundamental significance or not is currently being debated in the literature. We have nothing to add to this controversy other than to point out that even in the much simpler problem of NMR, actual quantitative calculations of $T_{2}$ are rather difficult because so many different processes could cause dephasing at low temperatures. In the $\tau_{\phi}$ problem, such dephasing processes include, for example, electron heating, magnetic impurities, background radiation, unknown inelastic channels at very low energies, finite system sizes, and actual slight movements of the impurity atoms which are considered to be static in the theory. Indeed, at low enough temperatures, where the dephasing time is already relatively long, any weak coupling to the environment will cause (weak) decoherence and lead to apparent saturation of $\tau_{\phi}$ at finite effective electron temperatures. 


\section{CONCLUSION}

We conclude by emphasizing that understanding dephasing and decoherence in specific solid state quantum computer architectures is crucial in the development of successful qubit operations in scalable solid state systems. For example, the Rabi oscillations observed [29] in the superconducting Cooper pair box system seem to have a very short dephasing timeof the order of nanoseconds only. Currently there is no theoretical understanding of such a short dephasing time in this system, although various electrical noise sources in the circuit could be one possible problem. Similarly, the anticipated flux qubitstates in the Josephson junction SQUID system [28, 30] have not yet demonstrated Rabi oscillations, perhaps because of strong decoherence or for other fundamental reasons. In semiconductor quantum dot based spin qubit systems the decoherence time $\left(T_{2}\right.$ or $T_{2}^{*}$ depending on the specific experiment) is experimentally measured to be in the $10 \mathrm{~ns}$ regime [44], which is far too short for any effective quantum computation operations$T_{2}$ in the range of $\mu \mathrm{s}$ to ms will be required for useful quantum computation. Theoretical estimates [41, 58 60] for relaxation times tend to concentrate on $T_{1}$, which is a conceptually simpler quantity to compute (being an inelastic relaxation time) $-T_{2}$ calculations must take into account the details of the specific experimental setup. In the context of TLS dynamics, it is important to emphasize that while $T_{1}$ refers to the lifetime of the excited $|1\rangle$ state (i.e. $T_{1}$ is the time over which the excited state $|1\rangle$ decays to the ground state $|0\rangle$ through the inelastic emission of photons, phonons, magnons, etc.), $T_{2}$ refers to the coherence of the superposition state $(|0\rangle+|1\rangle) / \sqrt{2}$ between the ground and the excited state and as such depends on the precise phase relationship between $|0\rangle$ snd $|1\rangle$ in a specific experimental setup where all the details of the experimental circuitry may matter in a nontrivial manner. This makes $T_{2}\left(\leq T_{1}\right)$ intrinsically more intractable than $T_{1}$ from a theoretical standpoint. We point out that quantum computation is possible in principle because single qubit quantum dephasing, while often being continuous in actual experimental situations, can always be equivalently represented as a discrete phase flipping process. Understanding dephasing in solid state quantum computer architectures (or in general, in solid state systems) remains one of the important problems in condensed matter physics.

\section{ACKNOWLEDGEMENT}

This work is supported by ARDA, DARPA, and ONR.

[1] M.A. Nielsen and I.L. Chuang, Quantum Computation and Quantum Information (Cambridge University Press, New York, 2000).

[2] W.H. Zurek, Phys. Today 44, 36 (1991).

[3] D. Loss and D.P. DiVincenzo, Phys. Rev. A 57, 120 (1998).

[4] G. Burkard, D. Loss, and D.P. DiVincenzo, Phys. Rev. B 59, 2070 (1999).

[5] X. Hu and S. Das Sarma, Phys. Rev. A 61, 2301 (2000).

[6] G. Burkard, H.A. Engel, and D. Loss, Fortschr Phys 48, 965 (2000).

[7] X. Hu and S. Das Sarma, LANL preprint cond-mat/0101102. To appear in Phys. Rev. A (2001); LANL preprint condmat/0102019.

[8] A. Imamoglu et al., Phys. Rev. Lett. 83, 4204 (1999); A. Imamoglu, Fortschr. Phys. 48, 987 (2000).

[9] T.A. Brun and H.L. Wang, Phys. Rev. A 61, 032307 (2000).

[10] P.M. Platzman and M.I. Dykman, Science 284, 1967 (1999); Fortschr Phys 48, 1095 (2000).

[11] C.H.W. Barnes, J.M. Shilton, and A.N. Robinson, Phys. Rev. B 62, 8410 (2000).

[12] R. Vrijen et al., Phys. Rev. A 62, 012306 (2000).

[13] S. Bandyopadhyay, Phys. Rev. B 61, 13813 (2000).

[14] R. Ionicioiu, Amaratunga, and F. Udrea, Int. J. Mod. Phys. B 15, 125 (2001); R. Ionicioiu, P. Zanardi, and F. Rossi, Phys. Rev. A 63, 050101 (2001).

[15] J. Levy, LANL preprint cond-mat/0101026.

[16] N.H. Bonadeo, J. Erland, D. Gammon, D. Park, D.S. Katzer, D.G. Steel, Science 282, 1473 (1998); G. Chen, Science 289, 1906 (2000).

[17] M.S. Sherwin, A. Imamoglu, T. Montroy, Phys. Rev. A 60, 3508 (1999).

[18] B.E. Cole, J.B. Williams, B.T. King, M.S. Sherwin, and C.R. Stanley, Nature 410, 60 (2001).

[19] M. Bayer et al., Science 291, 451 (2001). 
[20] P.C. Chen, C. Piermarocchi, L.J. Sham, Physica E 10, 7 (2001); Phys. Rev. Lett. 87, 067401 (2001).

[21] G.D. Sanders, K.W. Kim, and W.C. Holton, Phys. Rev. B 61, 7526 (2000); Phys. Rev. A 60, 4146 (1999).

[22] J.H. Reina, L. Quiroga, and N.F. Johnson, Phys. Rev. A 62, 012305 (2000).

[23] B.E. Kane, Nature 393, 133 (1998); Fortschr. Phys. 48 (2000), 1023.

[24] J.L. O'Brien et al., LANL preprint cond-mat/0104569.

[25] V. Privman, I.D. Vagner, and G. Kventsel, Phys. Lett. A 239 (1998), 141; D. Mozyrsky, V. Privman, and L. Glasser, Phys. Rev. Lett. 86, 5112 (2001).

[26] G.P. Berman, G.D. Doolen, P.C. Hammel, V.I. Tsifrinovich, Phys. Rev. B 61, 14694 (2000); Phys. Rev. Lett. 86, 2894 (2001).

[27] F. Yamaguchi and Y. Yamamoto, Appl. Phys. A 68, 1 (1999); J.R. Goldman, T.D. Ladd, F. Yamaguchi, and Y. Yamamoto, Appl. Phys. A 71, 11 (2000).

[28] A. Shnirman, G. Schön, and Z. Hermon, Phys. Rev. Lett. 79, 2371 (1997); D.V. Averin, Solid State Comm. 105, 659 (1998).

[29] Y. Nakamura, Y.A. Pashkin, and J.S. Tsai, Nature 398, 786 (1999).

[30] J.R. Friedman, V. Patel, W. Chen, S.K. Tolpygo, J.E. Lukens, Nature 406, 43 (2000); C.H. van der Wal et al., Science 290, 773 (2000).

[31] M.N. Leuenberger and D. Loss, Nature 410, 789, (2001).

[32] D.P. DiVincenzo, D. Bacon, J. Kempe, G. Burkard, and K.B. Whaley, Nature 408, 339 (2000).

[33] B.E. Kane et al., Phys. Rev. B 61, 2961 (2000).

[34] C. Cohen-Tannoudji, B. Diu, and F. Laloë, Quantum Mechanics (John Wiley and Sons, New York, 1977).

[35] C.P. Slichter, Principles of Magnetic Resonance (Springer-Verlag, Berlin, 1990).

[36] C.P. Poole Jr, Electron Spin Resonance (Interscience, New York, 1967).

[37] P. Meystre and M. Sargent III, Elements of Quantum Optics (Springer-Verlag, Berlin, 1999).

[38] F. Bloch, Phys. Rev. 70, 460 (1946).

[39] L.J. Sham, Journal of Magnetism and Magnetic Materials 200, 219 (1999).

[40] Optical Orientation, Modern Problems in Condensed Matter Science, Vol. 8, edited by F. Meier and B.P. Zakharchenya (North Holland, Amsterdam, 1984).

[41] J. Fabian and S. Das Sarma, J. Vac. Sci. Technol. B 17, 1708 (1999).

[42] M. Seck, M. Potemski, and P. Wyder, Phys. Rev. B 56, 7422 (1997); H.W. Jiang and E. Yablonovitch, Phys. Rev. B 64, R041307 (2001). See also, D. Stein, K. von Klitzing, and G. Weimann, Phys. Rev. Lett. 51, 130 (1983) for an early ESR measurement in GaAs heterostructures.

[43] J.M. Kikkawa and D.D. Awschalom, Phys. Rev. Lett. 80, 4313 (1998); A.P. Heberle, W.W. Rühle, and K. Ploog, Phys. Rev. Lett. 72, 3887 (1994).

[44] J.A. Gupta, D.D. Awschalom, X. Peng, and A.P. Alivisatos, Phys. Rev. B 59, R10421 (1999).

[45] J.A. Gupta, R. Knobel, N. Samarth, and D.D. Awschalom, Science 292, 2458 (2001).

[46] A.V. Khaetskii, Physica E 10, 27 (2001); S.I. Erlingsson, Y.V. Nazarov, and V.I. Fal'ko, LANL preprint cond-mat/0104148.

[47] T. Fujisawa, Y. Tokura, and Y. Hirayama, Phys. Rev. B 63, 081304 (2001).

[48] H.-A. Engel and D. Loss, Phys. Rev. Lett. 86, 4648 (2001).

[49] K.V. Kavokin, Phys. Rev. B 64, 075305 (2001).

[50] N.E. Bonesteel, D. Stepanenko, D.P. DiVincenzo, LANL preprint cond-mat/0106161; G. Burkard and D. Loss, LANL preprint cond-mat/0108101; R. Sriharsha, X. Hu, and S. Das Sarma, unpublished.

[51] J. Schliemann, D. Loss, A. MacDonald, Phys. Rev. B 63, 085311 (2001).

[52] X. Hu and S. Das Sarma, to be published.

[53] X. Hu, R. de Sousa, and S. Das Sarma, Phys. Rev. Lett. 86, 918 (2001).

[54] R. de Sousa, X. Hu, and S. Das Sarma, Phys. Rev. A (2001, in press); LANL preprint cond-mat/0103410.

[55] B.L. Altshuler, A.G. Aronov, and D.E. Khmel'nitskii, Solid State Commun. 39, 619 (1981).

[56] D. Belitz and S. Das Sarma, Phys. Rev. B 36, 7702 (1987).

[57] P. Mohanty, E.M.Q. Jariwala, and R.A. Webb, Phys. Rev. Lett. 78, 3366 (1997).

[58] J. Fabian and S. Das Sarma, Phys. Rev. Lett. 81, 5624 (1998); ibid. 83, 1211 (1999).

[59] J. Fabian and S. Das Sarma, J. Appl. Phys. 85, 5075 (1999).

[60] W.H. Lau, J.T. Olesberg, and M.E. Flatte, LANL preprint cond-mat/0004461. 